\title{
A Robust Fault-Tolerant and QoS Centric Routing Protocol for Mobile-WMSNs: FTQ-RPM
}

\author{
A. Ajina ${ }^{1 *}$ and Mydhili K. Nair ${ }^{2}$ \\ 'Department of Computer Science and Engineering, Sir M Visvesvaraya Institute of Technology, \\ Bangalore - 562157, Karnataka, India; ajinajaya@gmail.com \\ 2Department of Information Science and Engineering, M. S. Ramaiah Institute of Technology, \\ Bangalore - 560054, Karnataka, India; mydhili.nair@gmail.com
}

\begin{abstract}
Objectives: In this study we developed a routing protocol named Fault-Tolerant QoS Centric Routing Protocol for WMSN (FTQ-RPM) where the classical RPL routing protocol was augmented with mobility features and multiple network condition parameters based parent node selection for forwarding path selection. Methods/Statistical Analysis: Fault-resilient routing protocol for Low power Lossy Networks (LLNs) which is applied in parallel to the link layer that once detecting any link outage initiates node discovery. The use of Received Signal Strength Indicator (RSSI) and Expected Number of Control Packets (ETX) based best parent node selection makes FTQ-RPM to achieve fault-resilient routing decision. In addition, RSSI based mobility management or mobile node positioning makes data communication more reliable than the random mobility. It assures reliable data communication over mobile-RPL based WMSNs. FTQ-RPM applies a global link repair model and supplementary forwarding path selection that works in parallel to the link layer of the native-RPL. It assures timely data delivery through supplementary path without imposing any additional computational overheads, delay and energy exhaustion during any link-outage condition. Findings: The overall developed routing protocols have been examined in terms of throughput, real-time data delivery, packet loss, delay, power consumption, resource utilization etc, where the proposed systems have been found superior than state-of-art existing protocols. Application/Improvements: It affirms the suitability of the proposed routing protocols for real-time WMSNs applications particularly for IoT.
\end{abstract}

Keywords: Fault-Tolerant Routing, Low Power Lossy Networks, Mobility, Protocol, Quality of Service, WMSN

\section{Introduction}

In recent years, the exponential rise in the demand of wireless communication systems has been witnessed globally. The high pace rise in QoS and reliable communication demands have motivated academia-industries to achieve more efficient communication systems. Among varied existing communication systems, WSNs have been found potential solution to meet major demands including civil surveillance, industrial monitoring and control, defense application, healthcare sector and many more. The decentralized and infrastructure-less characteristics make WSN a potential solution. However, factors like the efficacy of WSN for multimedia data communication; mobility features etc motivate authors to achieve better solution.

In recent years, to perform low cost and efficient multimedia data communication Wireless Multimedia Sensor Networks (WMSN) has emerged as a promising technique. Undeniably, WMSNs can have significant potential

${ }^{*}$ Author for correspondence 
in real-time communication systems; however the use of classical WSN routing approaches suffers adversely in Low power Lossy Networks (LLNs) that eventually affects QoS provision, energy consumption and delay. It affects QoS provision, which has become an inevitable need for major LLNs applications.

Considering architectural aspects of WMSNs, it comprises multiple small sensors connected wirelessly to enable multimedia data acquisition and transmission to the base station for decision purposes ${ }^{1}$. Noticeably, multimedia data can be still images, audio, video and hybrid data. These novelties have made WMSNs to play vital role in IoT applications $s^{2,3}$. The efficacy of low-power technologies like 6 LowPAN ${ }^{4}$ towards IPv6 too encourages academia-industry to explore possibility of WMSNs to serve IoT applications, primarily multimedia broadcast over LLNs. In IoT multimedia communication, there is a streaming server that transmits multimedia data through object or application oriented devices and broadcasts it over wideband connections to the multiple users to avail subscribed services. In this process, the server is capable to exhibit computationally intensive processes including channel coding, encoding, transmission, etc. On contrary, decoder unit functions in slave mode for decoding the received data packets. Multimedia sensors deployed in WMSN undergoes various complexity and adversaries, such as bandwidth constraints, energy exhaustion, contention, resource allocation, and computation that make classical encoding limited for QoS centric applications. The sensor generated multimedia data often shows higher spatial correlation because of dense node deployment that demands certain efficient fault-resilient and timely data transmission protocol to ensure QoS delivery ${ }^{\underline{5-7}}$. A number of researches have been done to augment WSN routing protocols; however almost all efforts either focuses on energy optimization or the throughout optimization ${ }^{8-12}$. Considering present day application majority of the existing approached are confined and are suitable only for single target achievement.

Routing Protocol for Low power Lossy Network (RPL) was proposed by Internet Engineering Task Force (IETF) in $2008^{\frac{13}{3}}$ to meet QoS centric communication over LLNs ${ }^{14}$. LLN signifies a kind of network condition aware routing approach suitable for communicating under varying link conditions and unpredictable temporal and spatial network characteristics that often results into link outages and data loss. LLNs are defined in terms of high data loss, path loss, channel fading, multi-path interferences, low connectivity, link-outage probability, low power devices, etc. WSN being one of the dominating examples of LLNs too requires alleviating aforesaid adversaries to meet QoS demands. Undeniably, RPL based WSNs have been found potential for reliable data transmission; however its native routing approach doesn't consider any mobility feature that confines its applicability in LLNs. Mobility feature with sensor network could ensure cost-efficient communication over LLNs. Meanwhile, mobility with RPL for multimedia communication can be of paramount significance to meet WMSNs related demands. Most of the IoT applications employ mobility assisted communication and therefore enhancing native RPL with mobility can be vital for multimedia transmission ${ }^{\frac{13}{3}}$. Authors $\frac{15,16}{}$ has made effort to introduce mobility with RPL; however, majority of existing methods undergo link instability and responsiveness problem due to topological variations. In addition, dynamic topology of the generic routing might lead link loss and therefore significant packet loss. Consequently, it introduces delay and retransmission probability that eventually affect QoS delivery. On contrary, there are different applications where LLNs are supposed to provide QoS delivery.

Applications like multimedia data transmission requires higher degree of QoS provision for which ensuring fault-resilience is must. IEEE 802.15.4 MAC, which is a typically used link layer protocol in WSN possesses an ACK model to minimize data loss during link outage by performing retransmission; however it happens at the cost of energy exhaustion. Practically, the maximum retransmission count is $4-5$, in which the retransmission is scheduled at the interval of $10 \mathrm{~ms}$. Although, retransmission could exhibit Packet Delivery Ratio (PDR) successful for certain transient link outage for small period, it is inefficient in avoiding packet drop, especially in the situation when wireless channel has higher probability of node fail- 
ures. To avoid such adversaries, typically an alternate path selection approach is considered that facilitates a supplementary relay node to form alternative path towards destination to ensure successful delivery. Additionally, it could help in selecting an auxiliary next-hop node based on link quality to ensure reliable transmission. In RPL based LLNs, usually ETX and RSSI are applied as decision variable to assess link quality of a node to form forwarding path. However, very less effort has been made on introducing both these parameters to perform parent node selection and best forwarding path selection. In this paper, these two parameters are used to derive a link quality index for best parent node selection and forwarding path formation for QoS delivery over WMSNs.

In this paper a robust Fault-Tolerant and QoS Centric Routing Protocol for Mobile-WMSN over LLNs (FTQRPM) is developed for WMSN over LLNs. Unlike classical RPL routing approaches where merely default Objective Function (OF0) or ETX has been considered, FTQ-RPM applies dual objective functions ETX and ARSSI for parent nod selection and supplementary path formation. The proposed FTQ-RPM model is applied in parallel to the link layer that once detecting any link outage initiates node discovery and exploits ARSSI and ETX parameter to select best parent node and alternate forwarding path formation. Realizing the issues of dynamic topology we have performed RSSI adaptive mobile node movement control that helps avoiding link outage. It helps greatly in assuring QoS provision over WMSNs.

The other sections of the presented paper are divided as follows: Section II presents the related works, Section 3 briefs about the RPL routing protocol, it is then followed by problem formulation in Section 4 and proposed model discussion in Section 5. Results obtained are discussed in Section 6. The overall research conclusion and future scopes are discussed in Section 7 and the references used in this manuscript are presented at the end of the paper.

\section{Literature Survey}

This section discusses some of the key literatures pertaining to WMSNs routing protocol, RPL routing protocol, mobile WSN and RPL based WMSN routing protocol.
To ensure optimal performance over WMSNs, the key requirements are the optimal PDR, minimum data drop, low energy consumption and optimal bandwidth utilization ${ }^{1}$. Additionally, minimum end-to-end delay too is inevitable for WMSNs QoS provision. Authors suggested MAC optimization by incorporating cross-layer proto$\mathrm{col}^{17}$. $\mathrm{In}^{\frac{18}{8}}$ receiver based traffic scheduling was performed using a time-hoping impulse ratio model to enhance MAC. However, realizing the network dynamism, authors focused on handshaking based link formation to avoid sudden link outage. Authors $\frac{19,20}{}$ applied entropy adaptive data transmission rate for QoS centric routing.

They applied correlation between nodes to schedule data transmission; however multimedia sensors exhibit no significant spatial correlation with each other. Though cross-layer models apply multiple network statistics to obtain best forwarding node selection, authors ${ }^{20}$ derived it with transport, network and PHY to perform timeefficient data delivery. A relatively simpler model was developed in; however, it couldn't address the link adaptation concept to avoid link outage. To alleviate this issues, authors $\frac{21,22}{}$ applied adaptive link quality to perform route decision for WMSNs. Geographic routing protocols ${ }^{20,21}$ considers network states to identify best forwarding path. However, this approach considered relative distance between source and sink to form multiple paths for data transmission. $\mathrm{In}^{2 \underline{23}}$, multipath routing was proposed to ensure timely data delivery; however it was found effective only for short range communication with data rate of 50-250 kbps. However, major WMSNs require long range communication with higher data rate transmission to meet QoS needs. Varying the contention window size, authors ${ }^{24-26}$ enhanced MAC of the protocol stack. To ensure QoS, authors ${ }^{24,27}$ developed MAC optimization model using bandwidth and delay as decision parameter for best forwarding node selection. Some additional decision parameters were considered in ${ }^{\underline{28}}$. However, applying multiple parameters makes decision intricate and to resolve this issue authors ${ }^{29}$ applied evolutionary computing approaches. However, it could not address the mobility based routing problem. A few efforts were made in $\frac{30,31}{10}$ ensure minimal delay in real time multimedia transmis- 
sion over sensor networks. Though, these approaches have been developed for strengthening routing protocol stack to ensure timely and bandwidth efficient routing; however majority of these approaches are confined with LLNs. RPL routing approaches has been found a potential alternative, particularly for IoT environment with Lossy network conditions. However, the classical RPL doesn't have node-mobility feature. Realizing this gap, authors ${ }^{32,33}$ incorporated single mobile sink based routing. Since RPL based routing applies different control messages during network discovery and path formation, to reduce signaling overhead, authors ${ }^{34}$ developed a lowreactive route decision model for mobile-RPL. Though, not much significant works are done to incorporate RPL for multimedia data transmission, a few efforts are made to incorporate mobility with RPL over LLNs $\frac{35,36}{3} \operatorname{In}^{\frac{36}{}}$, mobility prediction approach was applied to enhance PDR; however, in real time application its efficacy seems limited. In ${ }^{37}$ made effort to augment network layer of RPL for higher PDR. $\operatorname{In}^{\frac{38}{}}$, focus was made on enhancing trickle timer optimization; however merely timers trickle can't assure fault resiliency under mobility. Though, a few efforts have been made on incorporating mobility based WMSNs, there is the scope for further optimization by applying network link adaptive forwarding path selection over LLNs. Applying mobility assisted RPL for WMSNs over LLN can play vital role in enabling QoS delivery in IoT ecosystem.

Though, the presented research work employs mobileRPL for multimedia data transmission over WMSNs and LLNs, briefing RPL routing protocol is must. Section III discusses the RPL routing protocol and associated terminologies.

\section{RPL - An Overview}

RPL protocol can be stated as a proactive routing approach particularly designed for communication or data transmission over LLNs, which makes it suitable for IoT ecosystem.RPL routing protocol functions based on the topological paradigm of Directed Acyclic Graphs (DAGs), where it functions by employing three key network parameters; DAG Information Solicitation
(DIS), DAG Information Object (DIO) and Destination Advertisement Object (DAO). Applying these control messages or attributes RPL forms Destination Oriented Directed Acyclic Graph (DODAG), which can be called as best forwarding node to transmit data packets. Noticeably, DAG signifies the default tree structure of RPL over LLNs. However, in practice it undergoes changes as per communication demands and topological variations. The basic topological attribute of RPL is DODAG that is formed at the node called DODAG root. RPL can have a number of DODAGs that could be defined in terms of the following attributes:

DODAGID: It signifies the root of DODAG.

RPLInstanceID: It recognizes certain autonomous set of DODAGs which could be augmented for particular condition.

DODAG VersionNumber: It augments the event like DODAG formation.

Rank: It signifies the position of a node in conjunction with the root node in a DODAG, where the individual node is provided a rank that increases in the downward direction of the DAG and vice versa.

In general, RPL applies different control messages to perform ND, link formation and data transmission. A snippet of these control messages are given as follows:

\subsection{RPL Control Messages}

RPL control messages are nothing else but a kind of Internet Control Message Protocol version 6 (ICMPv6) (RFC 2463), having four predominant types:

- DIO: It is invoked by DODAG root to constitute a new DAG and then the newly formed DODAG structure permits the multicast transmission. DODAG embodies key network information that helps node to exhibit RPL instance discovery; DODAG parent set construction and its maintenance. 
- DIS: It identifies the neighboring nodes in connected DODAGs.

- DAO: DAO transmits reverse route information to detect the nodes visited towards the "bottom to top" path. DAO control message is transmitted by each node in the network, except the DODAG root to maintain route tables containing information about the associated children nodes

- Destination Advertisement Object Acknowledgment (DAO-ACK): This control message is transmitted in the form of a unicast packet by a DAO recipient who transmits DAO message as the reply.

In process, the rank information of each node is transmitted through DIO in such manner that the sensor nodes placed farther from the root would possess higher ranks as compared to the nearer one. This feature play vital role in looping problem and hence eliminates the possibility of fault or delay. To form DODAG to enable data transmission, RPL applies a factor called OF that decides suitability of a node to become parent. In practice, OF is selected in such manner that it could enable QoS delivery. Typically, the default OF (i.e. OF0) and ETX are applied to assess suitability of a node to form forwarding path. DIO message functions to form bottom to top route, while DAO is unicast by the child nodes to their parent node so as to constitute top to bottom path. Though, the frequency of DIO message affects computational and signaling cost, RPL applies a trickle timer based scheduling to avoid unwanted DIO messages $\frac{35}{}$. The uses of trickle timer support early network convergence and reduces burden of additional control messages. To further optimize the computational efficacy of the network, deciding optimal interval of trickle timer can play vital role. Once any topological change takes place, trickle timer is reset which is then followed by ND in which the rank matrix of each node is obtained and its suitability to become parent node is examined to ensure reliable data transmission.

\section{Problem Formulation}

To achieve QoS centric communication over mobileRPL based WMSNs, ensuring optimal best parent node selection and best forwarding path formation are vital. Though, the main issue in mobile-RPL is mobility and resulting topological changes that increases link outage probability, which is not appropriate for multimedia data transmission. Especially for IoT ecosystems, ensuring reliable and QoS centric data transmission is must. To deal with such issues, typically researchers prefer applying multipath forwarding approach by incorporating packet-replication over different links. Though, these classical approaches can help in reducing latency and network convergence, however the probability of link-outage remains an open research area. Major existing works employs higher control packets and associated signals that impose significantly high computational overheads and energy consumption. On contrary, the presence of mobility introduces network (link) uncertainty among nodes (say, RPL DODAG) that makes routing and data transmission highly uncertain, particularly for multimedia data transmission which requires timely and resource efficient transmission. Though, the enhancement of RPL with mobility and multimedia transmission can be vital our research work intends to incorporate multiple value additions, including link quality adaptive mobility management, dual objective function based link quality assessment, best forwarding node selection, fault-resilient supplementary path formation for reliable and QoS centric data transmission. Majority of the existing mobile-RPL routing protocol for IoT applications uses default OF0 to perform best parent node selection and DODAG formation. However, the varying network conditions and allied parameters changes, like RSSI, inter-node distance etc affect link-reliability and performance. To alleviate these issues timers like connectivity timers, response-tracking timers, handoff management timers, etc can be applied to continuously monitor link quality so as to assist reliable forwarding path selection. However, it can introduce computational overheads.

Mobility based RPL for LLN undergoes topological changes continuously that might cause link outage. To 
alleviate such issues, the standard RPL embodies linkoutage repair methods such as local link repair and global link repair. In practice, the local link repair model is executed once the parent node undergoes fault conditions. On the contrary, the global repair model is executed by the parent node once the network is re-constituted by means of a modified DODAG to further network reconstruction. Link level local repair comes in function once a parent node undergoes adversaries that night be normal in case of dynamic topology variation. To ensure reliable and QoS centric communication over WMSNs, detecting parent node failure and selecting an alternate parent from parent set can be vital. In our proposed routing model, once detecting link outage, at first the root node identifies the best parent node available in parent node set. Noticeably, based on respective node rank and key parameters (ARSSI and ETX), nodes are updated in a parent node set.

The nodes are updated in node table based on decreasing order of the rank matrix. When detecting link outage, the proposed model at first identifies the best parent node to be used for supplementary path formation, which is then followed y DODAG formation and data transmission over WMSNs. If there is no alternate parent node available in parent node set, the root node could behave as a new node and executes Node Discovery (ND) where it transmit DIS message to obtain best parent node of the forwarding node.

Link-outage forces network to initiate ND and alternate path formation that as a result imposes huge control packets overhead, delay and energy exhaustion. In addition, it reduces PDR and hence influences overall QoS delivery. Considering these problems, in this paper a novel fault tolerant mobile-RPL routing protocol has been developed for multimedia data transmission over WMSNs. Unlike traditional approaches, FTQ-RPM applies multiple network parameters such as ARSSI and ETX as the objective functions to assess suitability of anode to become parent node for forming forwarding path. Considering resource utilization aspect of WMSNs, once receiving ACK from the receiver node, the backup data is deleted that makes process resource efficient. To ensure backward compatibility of RPL, FTQ-RPL functions in parallel to the network layer, where the parent nodes are ordered on the basis of respective link quality index such as ETX and ARSSI. One of the dominating factors affecting performance is the MAC retransmission limit. In fact, it plays vital role in deciding the time for ND to obtain best forwarding node and path formation. Additionally, the calculation of ARSSI (it signifies the average RSSI which is obtained through DAO message obtained from each node after DIS multicast from the root node) to be used in estimating PDR prediction ensures that the data would be transmitted successfully. In fact, higher ARSSI signifies higher PDR, which is must for WMSNs. In our model, we have also applied ARSSI to estimate ETX count required to transmit data to the sink that makes decision process more efficient to ensure time and computation efficient transmission. In addition to the above stated novelty, to further strengthen the fault resiliency, in this paper we have exploited ARSSI information to decide mobile node mobility. Here, estimating the ARSSI of each node, the mobile node is positioned to ensure maximum possible data gathering without causing any link outage or QoS violation. Thus, the overall proposed approach enables a highly robust and fault-resilient communication system for mobile-RPL assisted WMSN for multimedia data transmission.

\section{Our Contribution}

Considering the demand of a robust and efficient mobile-RPL based routing protocol for multimedia data transmission over WMSNs under LLN conditions; in this paper three predominant contributions have been made. These are:

- ARSSI based Mobile-node localization for reliable and fault tolerant data collection,

- Multiple Network Link Quality Indices based Best Forwarding Node selection and DODAG formation, and

- Fault-tolerant supplementary link formation for QoS assurance in WMSN over LLNs. 
Considering overall research contribution and novelties, we introduced a highly robust Fault-Tolerant and QoS Centric Routing Protocol for Mobile-WMSN over LLNs (FTQ-RPM) has been developed. A detailed discussion of the proposed method and its implementation is given as follows:

Once detecting any link outage or network failure during packet transmission, FTQ-RPM routing protocol is executed that functions in parallel to the IEEE 802.15.4 network layer to forward the same packets to the parent node. In our model, it has been accomplished by means of a best forwarding node selection or best parent node selection in the same DODAG. Noticeably, in FTQ-RPM the best parent node selection takes place by executing ND phase once any link outage is observed. Here, ND process continues till obtaining the best parent node to form alternate forwarding path for QoS delivery. During ND, the use of ETX and ARSSI ensures optimal best forwarding node selection, as maintaining minimum number of ETX supports minimum signaling overheads and energy consumption. On the other hand, higher ARSSI signifies better signal strength and hence higher PDR probability. The cumulative consideration of these two conditions ensures that the selected node as parent would ensure optimal PDR and timely data delivery without imposing any fault-probability or link-outage, even under dynamic topological conditions. Interestingly, RSSI value refers higher PDR probability, where PDR itself is related to the ETX counts. Therefore estimating ARSSI dynamically to maintain link reliability and minimum ETX can be of great significance. In our proposed model, a parent table is used that contains the set of parent nodes in decreasing order of their rank value (where rank is obtained based on ETX and ARSSI). Thus, re-structuring alternate

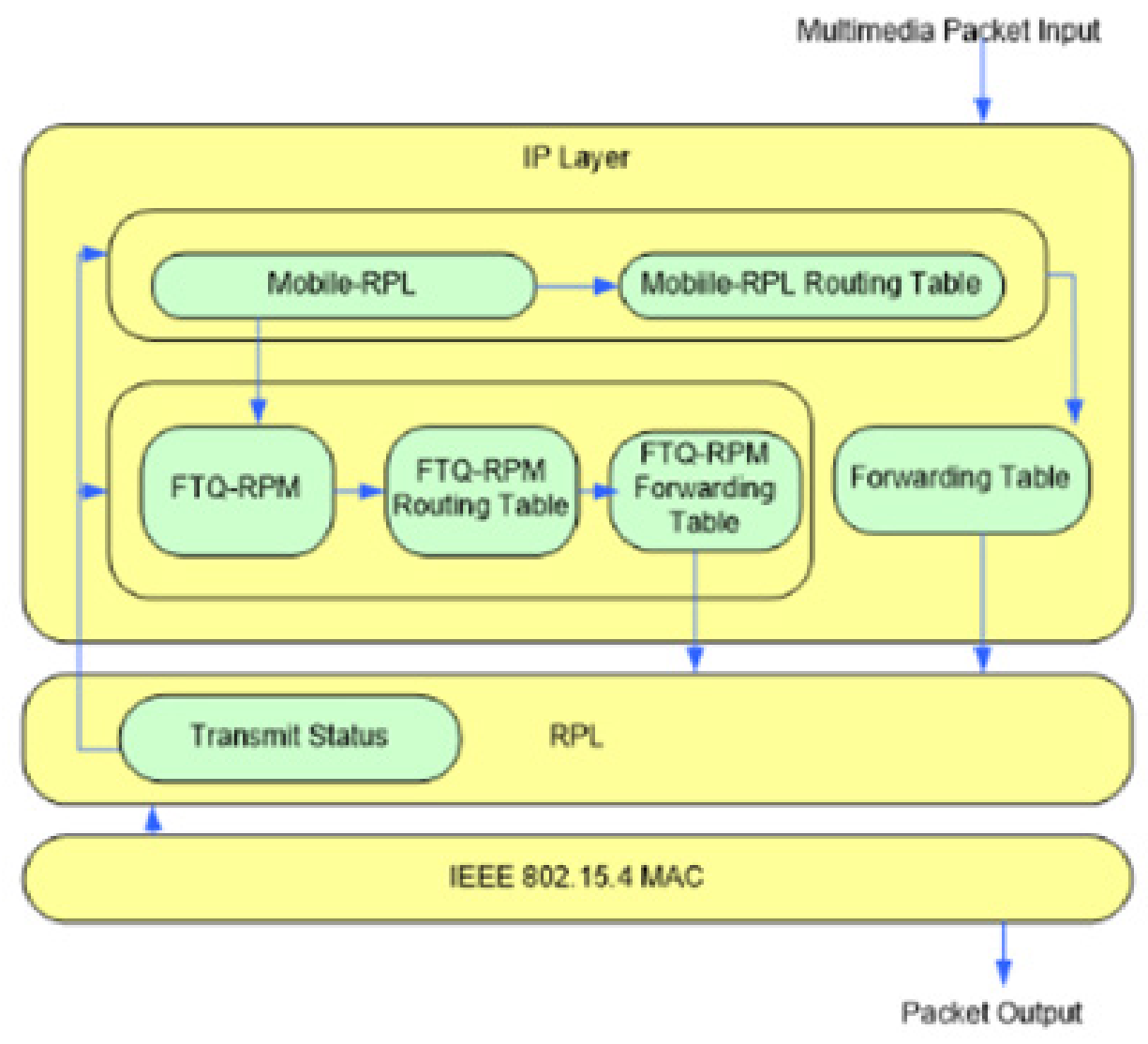

Figure 1. MALQI based packet forwarding in mobile-RPL. 
path, the data is transmitted from source to the destination node while alleviating fault-probability and delay. Figure 1 depicts a snippet of the overall functional model of the proposed FTQ-RPM protocol. This figure depicts the IP layer and its connectivity with the link layer of the mobile-RPL for WMSNs over LLN.

As already stated, unlike classical RPL routing models for LLNs, FTQ-RPM applies mobility feature to augment data gathering efficiency and signaling overhead reduction. Realizing the fact that the standard (native) RPL doesn't have any in-built mobility function and hence it requires a well defined approach to deal with mobility caused adversaries such as changes in network topology.

To ensure QoS centric communication fault-resilient mobility management is must. With this motive in our research we have derived a novel node positioning approach that exploits ARSSI information for mobility management. The proposed routing model is developed with both static sensor nodes as well as mobile sink node. A snippet of the communication between the static node (say, anchor node) and mobile node is discussed in the following sub-section.

\subsection{ARSSI based Mobile-node Localization for Reliable and Fault Tolerant Data Collection}

To ensure QoS delivery proper node localization often plays vital role, especially in case of mobility based sensor networks. With this motivation, FTQ-RPM unlike genetic random mobility or waypoint mobility, we have exploited dynamic network condition to perform mobile node localization that helps in avoiding any link outage probability. In this paper, Confidence Region (CR) concept has been applied for mobility management. A brief of our proposed node deployment strategy is given as follows:

\subsubsection{CRbased Node Localization}

As stated, FTQ-RPM applies both static node (i.e., anchor node) as well as mobile node. Here, we intend to deploy nodes in such way that even under mobility conditions it could not lead link outage. It not only avoids link outage probability but also supports fast network parameter measurement and routing decision. To further strengthen it, the dynamic mobile node positioning can be performed. In our work, the mobile node applies ARSSI of an anchor node to decide whether to travel towards that node to perform (forwarding) link formation or not. Since, in FTQ-RPM at ND phase ARSSI of each anchor node is obtained, which is exploited further to control mobile node movement, where mobile node intends to connect with the node having higher RSSI value. To achieve mobile node localization, we have derived CR, in which the mobile node decides its position by means of overlapping CR of the different anchor nodes. Unlike least square based node positioning CR based scheme performs characterization of the affect of each anchor node on link formation and data transmission. Undeniably, the RSSI value obtained for an anchor node located nearer to the mobile node would be often higher RSSI than the node located at relatively farther distance. It can have impact on mobile node positioning. To deal with such adversaries, in this paper we estimated CR for which it is considered that the mobile node is aware about the time-varying channel conditions following log-distance path loss model. Thus, the RSSI estimated at certain time instant $\mathrm{k}$ is:

$$
\operatorname{RSSI}^{k}=P_{1 m}-10 \alpha \log _{10} d-\gamma
$$

Where $P_{1 m}$ signifies the received signal at the distance of 1 meter (from the source node). The other variable $a$ signifies path-loss exponent and the radio range is $d$. The zero mean Gaussian noise is $\gamma \sim \mathcal{N}\left(0, \sigma_{Y}^{2}\right)$. Thus, the distance between mobile node and the anchor node is obtained as:

$\hat{d}_{R S S I}^{k}=10^{\frac{R S S I^{k}-p_{1 m}}{10 \kappa}}$

Noticeably, $\hat{d}_{R S S I}^{k}$ applies a Gaussian distribution approach due to the exponential associations between 
distance and RSSI values. A log-normal distribution is applied to estimate the inter-node distance, given by

$$
\ln \hat{d}_{R S S I}^{k} \sim \mathcal{N}\left(\ln d^{k}, \sigma_{d}^{2}\right)
$$

Where $d^{k}$ signifies the inter-node distance during kth time-slot. The standard deviation in distance calculated is $\sigma_{d}=\left(\sigma_{\gamma} \ln 10\right)=(10 \alpha)$. Applying these attributes, the Probability Density Function (PDF) is obtained as:

$$
f d(\hat{d})= \begin{cases}\frac{1}{\sqrt{2 \pi} \sigma_{d} \hat{d}} \exp -\frac{(\ln \hat{d}-\ln d)^{2}}{2 \sigma_{d}^{2}} \text { if } \hat{d}>0 \\ 0 \quad \text { if } \hat{d} \leq 0,\end{cases}
$$

In Equation (4), signifies the distance estimated. In this manner, obtaining PDF of the distance measure, the $\mathrm{CR}$ range of a-th anchor node is obtained. The variable $\mathrm{C}_{\mathrm{a}}$ states CR within which the mobile node is located with the confidence probability $\mathrm{q}_{\mathrm{LN}}$. Typically, major antennas used are the omni-directional antennas, signifies a circular periphery having radio range centered over -th anchor node location, with the conditions:

$$
\operatorname{Pr}\left\{d^{k} \leq r\right\}=q L N
$$

We have estimated $q L N$ using Equation (6).

$$
q L N(\mathrm{r}) \simeq \int_{0+}^{\mathrm{r}} \frac{1}{\sqrt{2 \pi} \sigma_{d} x} \exp \left(-\frac{\left(\ln x-\ln \hat{d}_{\text {RSSI }}^{k}\right)^{2}}{2 \sigma_{d}^{2}}\right) \mathrm{d} x
$$

Here, $\mathrm{m}$-th mobile node defines associated CR by performing overlapping of the CRs of the L closest anchor nodes. In other way,

$$
C_{m}=C_{1} \cap C_{2} \cap \ldots \ldots \cap C_{L^{\prime}}
$$

The mobile node calculates its position, $\widehat{P} m$ within CR by calculating the discrete points distributed within $\mathrm{C}_{\mathrm{m}}$. In our approach, the mobile node estimates the likelihood to be present in each point of $\mathrm{C}_{\mathrm{m}}$ by means of exploiting PDF. Let the PDFs of varied anchor nodes are autonomous; the mobile node calculates its position by Equation (8).

$$
\widehat{\mathrm{P}} m=\max _{\sigma} \prod_{a \in A^{P}} q L N\left(d_{c, a}\right) \forall c \in C_{m}
$$

Where $A^{v}$ signifies the anchor nodes functions in the process of mobile node positioning. In Equation (8) $\mathrm{d}_{\mathrm{c}_{j} \mathrm{a}}$ signifies the Euclidean distance a-th and c-th anchor node.

\subsubsection{Multiple Network Link Quality Indices based Best Forwarding Node Selection and DODAG Formation}

The overall mechanism of the data transmission of our proposed Mobile-RPL assisted QoS Centric Routing over WMSNs can be visualized in two steps; Inter-anchor node transmission and mobile-to-anchor node transmission. A snippet of these data transmission methods is given as follows:

Inter-anchor node transmission takes place between the anchor nodes. Since, this process doesn't involve mobile node and therefore there is no probability of link outage. In such cases the standard RPL based approach can be applied to communicate amongst (static) anchor nodes. To perform forwarding link formation, DODAG is constructed using DIOs messages. To form forwarding link, at first node transits a multicast message to all the nodes and receives DAO message as unicast from each neighboring node. The received packets as acknowledge- 
ment embody the key information such as ETX count and the ARSSI which is then used to estimate the rank of that node. Thus, based on respective node's rank a parent node is selected where a parent node set is formed which is updated proactively. In parent node set, the nodes are prioritized based on respective rank value. Thus, obtaining the best parent node the forwarding link is formed to perform multimedia data transmission. The anchor node $i$ estimates ARSSI of the node in the parent node table, based on which the PDR is estimated. Eventually, exploiting PDR value the ETX is obtained as follows. The end-to-end ETX number from the source $i$ and jth forwarding node is retrieved as ETX $_{\mathrm{i}, \mathrm{j}}$. Mathematically,

$$
E T X_{i}=\frac{1}{P D R_{i_{i j}}}+E T X_{j}
$$

In Equation (9), ETX $\mathrm{I}_{\mathrm{I}}$ signifies the overall ETX needed to reach destination node. ETX $_{\mathrm{j}}$ presents the total number of control messages (ETX) required from the jth node. Thus, the total ETX required between $i$ and $j$ can be obtained as the reciprocal of PDR (i.e. $\left(E T X_{i j}=\frac{1}{P D R_{i, j}}\right)$ ). In FTQ-RPM, ARSSI value

(which is obtained from the DIO messages obtained from unicast messages transmitted from the anchor nodes) is used to estimate rank value of the node. It strengthens FTQ-RPM to exhibit both reliable and least time-consuming and signaling cost approach for multimedia communication over WMSN. In addition to inter(anchor/static)-node communication, we have incorporated mobile node for swift and delay tolerant communication. However, the standard RPL doesn't consider any routing strategy for transmission under mobile topology ${ }^{39}$. To alleviate it, FTQ-RPM routing at first tries to perform link quality (i.e., RSSI) based mobile node localization, which is then followed by the use of ARSSI and ETX based parent node selection. This as a cumulative solution enables optimal routing over WMSNs. To perform link formation, the mobile node uses parent node from the parent-node set which are contained in the table based on rank values. When transmitting data packet from th anchor node, the total ETX required for th mobile node (say, ETX $_{\mathrm{m}}$ ) is obtained by adding ETX needed from the node itself with the th anchor node $\left(\right.$ ETX $\left._{\mathrm{m}_{t} \mathrm{a}} \mathrm{a}\right)$.

$$
E T X_{m}=E T X_{m, a}+E T X_{a}
$$

ETX $_{\mathrm{a}}$ may be obtained by exploiting the rank information of the th anchor node (9). Through, DIO which is transmitted from each node transmits acknowledgement message containing ETX and ARSSI information, it becomes easy to assess suitability of that access node to become the parent node. We have estimated PDR as the probability of successful packet transmission at the th time slot with the ARSSI more than the transceiver's sensitivity $\left(\mathrm{RSSI}_{\mathrm{th}}\right)^{40}$. In other way,

$$
P D R_{m_{i} a}=\operatorname{Pr}\left\{R S S I_{m_{l} a}^{k} \geq R S S I_{t h}\right\}
$$

With decreasing ARSSI over distance, the $\mathrm{PDR}_{\mathrm{m}, \mathrm{a}}$ is:

$$
P D R_{m_{i} a}=\operatorname{Pr}\left\{\gamma \leq P_{1 m}-10 \log _{10} d_{m_{i} a}-R S S I_{t h}\right\}
$$

Since, $\gamma$ signifies Gaussian distribution $\mathcal{N}\left(0, \sigma_{Y}^{2}\right)$, the PDR between access node and the mobile node is PDR $_{\mathrm{m}, \mathrm{a}}$ obtained as:

$P D R_{m_{i} a}=\operatorname{Pr}\{\gamma \leq X\}=\int_{-\infty}^{X} \frac{1}{\sqrt{2 \pi} \sigma_{y}} \exp \left(-\frac{\gamma^{2}}{2 \sigma_{y}^{2}}\right) d \gamma_{,}$ 
Here, the factor $\mathrm{X}$ is applied to ensure clarity rather than $\mathrm{P}_{1 \mathrm{~m}}-10 \alpha \log _{10} \widehat{\mathrm{d}}_{\mathrm{m}_{\mathrm{r}} \mathrm{a}}-\mathrm{RSSI}_{\text {th }}$. Here, $\mathrm{d}_{\mathrm{m}_{\mathrm{f}} \mathrm{a}}$ is substituted by $\widehat{\mathrm{d}}_{\mathrm{m}, \mathrm{a}}$, and therefore applying ARSSI value and allied PDR, mth mobile node $\mathrm{a}_{\mathrm{m}}$ performs parent node selection by:

$$
a_{m}=\arg \min _{k}\left(\frac{1}{P D R_{m_{k} k}}+E T X_{k}\right), \quad \forall k \in \mathcal{K}_{m^{*}}
$$

\subsubsection{Fault-Tolerant Supplementary Link Formation for QoS Assurance in WMSN over LLNs.}

For static nodes based communication, in RPL a node estimates the total number of ETX and ARSSI values. Based on these two factors it decides the parent node. Practically, a node exploits DIO message to retrieve dynamic link information of neighbor node to select parent node. Taking into consideration of the mobility in RPL based WMSN where there is continuous topological variation. The mobile nodes introduce link instability temporally as well as spatially, and hence assessing link quality of a node continuously is must. Additionally, on the basis of IoT application environment there can be all nodes as mobile over a defined network or mobile nodes could be connected to the relay node. In dynamic network condition, node may not have accurate network information; particularly the link quality because even parent node might be moving and hence associated network states could change spatially or temporally. The varying topology makes parent node selection tedious. The predominant reason behind it is the minimized DIO frequency that significantly reduces the suitability of a node to become parent node. Consider that though a node performs ND, since it hasn't sent packets to any of the parents available in parent set, estimating ETX or ARSSI is of no significance for best parent node selection. In fact, there is the dual purpose of applying dual objec- tive function (i.e. ETX and ARSSI). As ARSSI signifies the current link quality which is directly related to the PDR that it is related to the total number of ETX ${ }^{41}$, its use assures reliable link formation and multimedia data transmission over WMSNs. As ARSSI is obtained directly from the incoming DIO message that contains rank information enables selection of the path having minimum link-outage probability. Based on the DIO messages for the current link information the trickle timer can be disabled ${ }^{34}$. With this motivation, we disabled the trickle timer and hence DIO is transmitted at a predefined interval. We have assigned interval at 2 second.

To ensure QoS data transmission FTQ-RPM assures that each node in DODAG contains parent set which is updated proactively based on respective rank value. Additionally, it considers IEEE 802.15.4 protocol stack that provides needed connectivity at the network layer to obtain link layer information. Once sensing any link outage because of the node mobility, the link layer initiates FTQ-RPM in parallel. Initially, FTQ-RPM identifies the parent node from the parent node sets. Selecting the best parent node, FTQ-RPM forms a supplementary link as the best forwarding path to transmit data packets to the destination. If there is no best parent node available, FTQ-RPM initiates' ND in which the node transmits a beacon message as multicast to obtain the link information and node status, based on which it estimates the best parent node. Additionally, the other nodes in the decreasing order of rank value are stored in parent set. It reduces computational or signaling cost significantly. Thus, obtaining the best parent node a supplementary forwarding path is formed to transmit backup packet to the destination without imposing higher retransmission and delay. Once completing data transmission, the backup buffer is cleared to accommodate other packets for further transmission. A brief of the proposed routing protocol is given as follows:

Algorithm 1: FTQ-RPM Routing Protocol for WMSN 


\section{Algorithm 1 FTQ-RPM Routing Protocol for WMSN}

1: Packet ${ }_{\text {Cur }}$ : Multimedia packet for transmission.

2: Parent ${ }_{\text {Cur' }}$ : Current packet's receiving node.

3:Assign MAC $_{\left(\mathrm{N} \_R e T x_{\mathrm{R}}\right)}$ It defines the total number of MAC level retransmission of the current packet Packet $_{\text {Cur }}$ for Parent ${ }_{\text {Cur }}$

4:Choose the best alternate parent node parent ${ }_{\text {Alternate () }}$ for supplementary path formation and data transmission.

5:Obtain best parent node based on respective rank value or matrix parents ${ }_{\text {MaxRank }(0)}$

6: for each multimedia packet do

7:Set current parent as the default parent Parent ${ }_{\text {Cur }}$

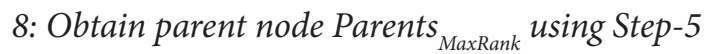

9:Initialize a matrix for attempted node for being parent node parent ${ }_{\text {attempted }}=0$

10: Prepare a backup data for current packet Packet ${ }_{\text {Cur }}$

11: Start packet transmission $T_{\dot{x}}$

12: Allocate $M A C_{(\text {retry count) }}\left(\right.$ Parent $_{\text {Current }}$ Packet $\left._{\text {Current }}\right)$

13: Transmit current data packet $\left(\right.$ Packet $\left._{C u r}\right)$

14: Parent node attempted++

15: Wait till receiving ACK $\left(M A C T_{\text {xstatus }}()\right)$

16: if $M A C T_{x s t a t u s=1}$ then

17: Clean buffer or delete backup data

18: Buffer ${ }_{\text {Backup }}()$, else

19: Update Parent $t_{\text {Cur }} \leftarrow$ Parent $t_{\text {Alternate }}$

20: if $\left(\left(\right.\right.$ Parent $\left._{\text {Current }} !\right) \|\left(\right.$ Parent $_{\text {attempted }}>$ Parents $\left.\left._{\text {MaxRank }}\right)\right)$ then

21: Clean buffer or delete backup data Buffer ${ }_{\text {Backup }}$

22: end if

23: Start packet transmission $T_{x}$

24: end if

25: end for. 


\section{Results and Discussions}

In last few years, wireless communication systems have gained immense significance to meet rising broadband communication demands. The increasing variations in use patterns, mobility conditions and network environment have always been a challenging issue for academia-industry to achieve a more efficient communication solution, particularly for QoS centric communication purposes. Undeniably, the increase in multimedia data transmission over wireless media has given a broad dimension to the WMSNs. However, to provide better employability of WMSNs for real-time multimedia data transmission QoS delivery is must that requires higher Packet Delivery Ratio with minimum or negligible delay and optimal bandwidth utilization. On the other hand, introducing mobility with WMSNs can provide a better alternative for low cost multimedia data transmission. However, the mobility in WMSN might introduce higher topological variation that as a result could vary network parameters. It may turn out to be more adverse in LLN conditions. Under such conditions ensuring reliable data transmission is a tedious task. To alleviate such issues, managing mobility (proactively) with certain enhanced routing approaches such as RPL and ensuring reliable communication can be vital. With this motivation, in this paper a novel routing protocol called Fault-Resilient and QoS centric Routing Protocol for WMSNs under LLN condition (FTQ-RPM) was developed. FTQ-RPM applied dynamic network parameters such as ETX and ARSSI to select the best parent node during network discovery so as to ensure reliable forwarding link formation. In addition, to deal with dynamic topology conditions, the mobile node exploited RSSI value of a node to perform localization. This enabled both link assurance as well as efficient data gathering. To ensure reliable and timely multimedia data delivery, FTQ-RPM was applied in the parallel of RPL Link payer that once detecting link outage at first tries to use alternate parent node from the parent set, which were ordered in the decreasing order of RSSI value and ETX required. Thus, applying the alternate parent node the root node forms an alternate forwarding path and thus transmits the backup data to the destination. In this manner the multimedia data is transmitted to the destination without introducing any significant delay and retransmission. It helps in enabling QoS delivery over WMSNs. The proposed routing protocol was developed based on RPL concept which was applied over IEEE 802.15.4 standard. The proposed routing model was developed in such manner that it retains backward compatibility that supports real time applicability in IoT ecosystem. To perform simulation, we took two types of nodes static anchor nodes and mobile nodes. For sake of simplicity we considered eight static node and one mobile sink node. Though, in simulation we varied the node density to examine performance under varying network size conditions. The RPL motes were taken as Sky-mote type. Here, we assigned trickle timer interval as $2 \mathrm{~ms}$. It alleviates the need of additional timers and unwanted node discovery phase that reduces signaling overhead incurred during control message transmission. The transmission model selected was UDGM.

FTQ-RPM protocol was simulated over ContikiCooja with Version InstantContiki 2.5 version. Though the proposed routing protocol was expected to be developed for multimedia data transmission over WMSN, realizing the (burst) transmission characteristics, the performance was examined by varying the packet transmission rate. The overall performance was examined in terms of PDR, node density Vs throughput, delay etc. To present the results in a well defined manner MATLAB tool was applied. To examine the performance of our proposed FTQ-RPM routing protocol, we have compared with a reference work done $\mathrm{in}^{42}$, where authors have applied Contiki simulation based on classical CSMA-CA routing protocol for multimedia data transmission. In the existing work ${ }^{42}$, authors have focused only on reducing collision probability and fair resource estimation. However, for multimedia data transmission in addition to the resource allocation avoiding any fault probability is also vital. Our proposed FTQ-RPM model considers it and exhibits better performance. On the other hand, in the existing system only one parameter ETX has been applied to select parent node and in addition it doesn't 


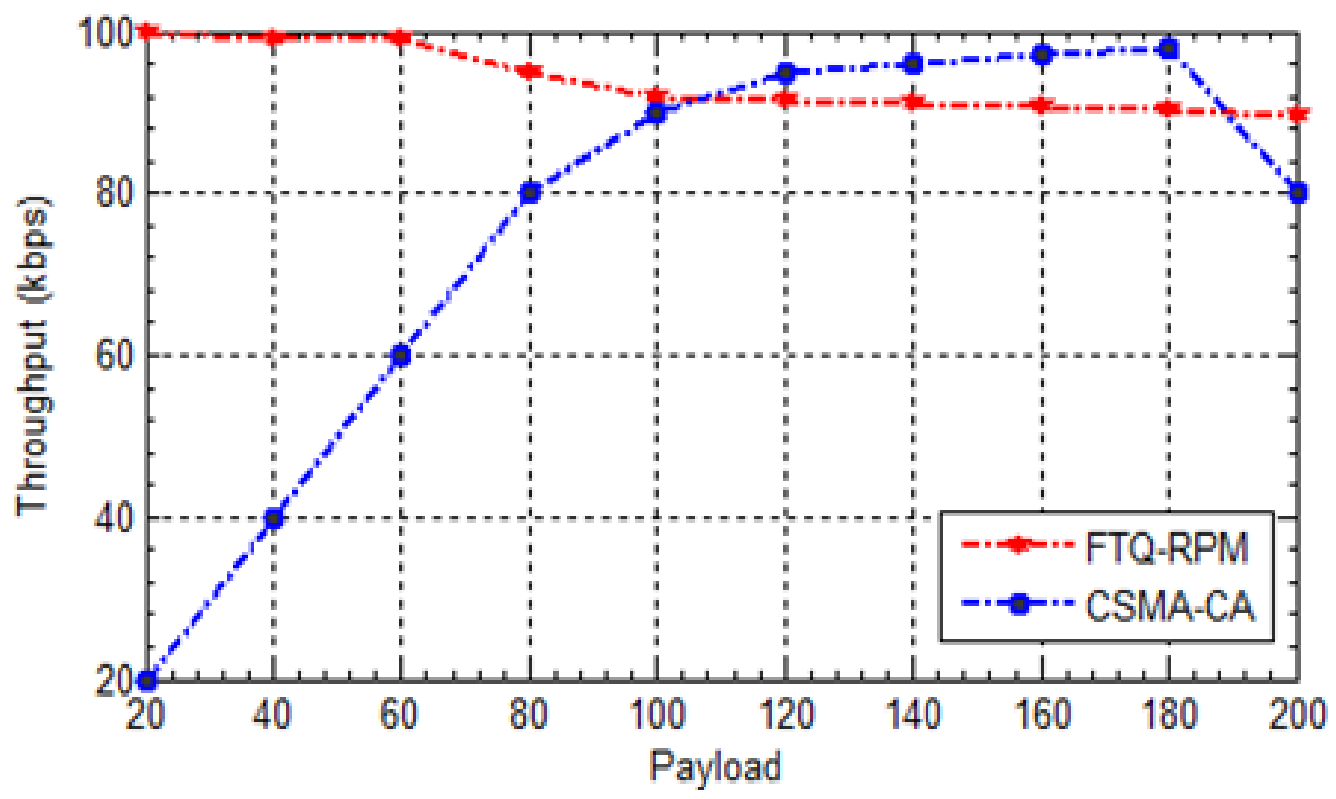

Figure 2. Throughput variation with payload.

consider any mobility assisted routing. Thus, comparing our proposed method with CSMS-CA based multimedia transmission; our proposed system is more robust.

Considering the fact that multimedia data sources such as audio, video and image data can have the non-uniform data transmission pattern. Under such circumstances strengthening a routing protocol to deliver higher throughput or PDR under varying payload conditions or transmission rate is must. As increase in payload and its successful delivery to the receiver is directly related to QoS of the multimedia transmission, we have examined our proposed FTQ-RPM routing protocol by varying payload condition. To assess the throughput of the proposed model we transmitted payload with varying packet size $20,40,60,80,120,160,180$ and 200 . The throughput performance by our proposed FTQ-RPM routing protocol is presented in Figure 2. To compare the performance, we have applied standard CSMA-CA protocol. Observing the result, it can be easily found that our proposed FTQRPM routing approach outperforms CSMA-CA routing. Since, CSMA-CA emphasizes only on collision avoidance and resource utilization, it doesn't consider link outage conditions that influences overall throughput delivery, as due to mobility there used to be the probability of data drop that consequently might take time for CSMA-CA based routing to obtain the best (alternate) parent node for forming best forwarding path selection. On the other hand, our proposed FTQ-RPM routing protocol applies multiple network link parameters to ensure that even due to mobility the link outage is avoided and therefore there would be the minimum drop probability. This as a result augments throughput, as depicted in Figure 2.

In Figure 3, the PDR variation with respect to the number of retransmission is presented. As depicted in result, the PDR performance is better for the proposed FTQ-RPM routing protocol. Since, our proposed routing model avoids link outage and therefore the number of retransmission is not much needed. On the other hand, the classical CSMA-CA routing protocol might undergo retransmission because of lack of link-outage (say, fault) resilience, and hence might be forced to perform retransmission. Undeniably, increase in payload affects the routing decision as in practice there could be the node fulfilling decision criteria; however due to low buffer could not be able to transmit the data. To alleviate this issue, our proposed routing model embodies both 


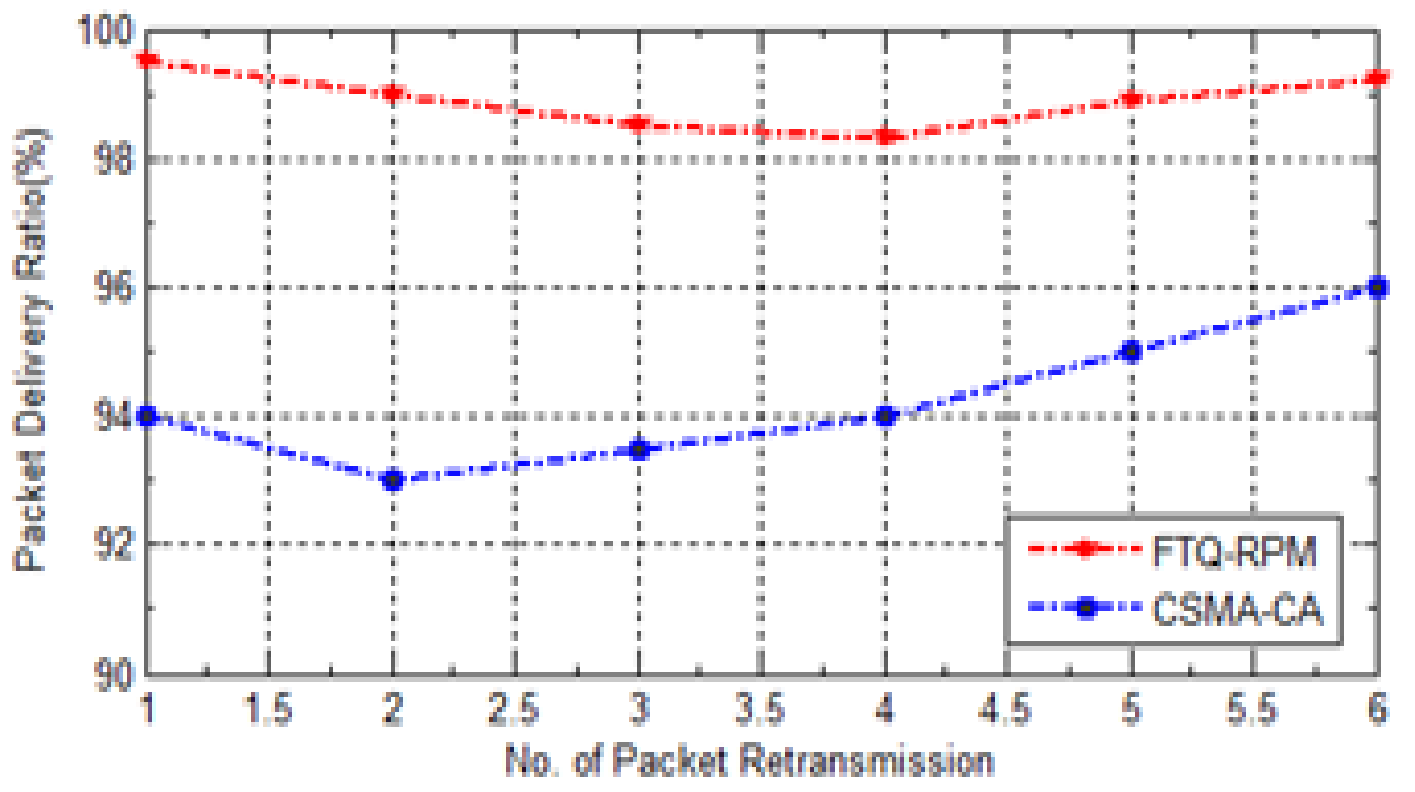

Figure 3. Packet Delivery Ratio (PDR) variation with increase in the number of retransmission.

native RPL as well as FTQ-RPM, which works in parallel of RPL's link layer.

This combination assures that the node doesn't undergo overloading condition or contention by exploiting efficacy of CSMA-CA in native RPL itself. It makes our proposed routing protocol robust to avoid any contention or congestion condition.

Figure 4 presents the packet loss with the increase in the number of control packets (i.e. ETX). As already stated to ensure timely communication which is inevitable for

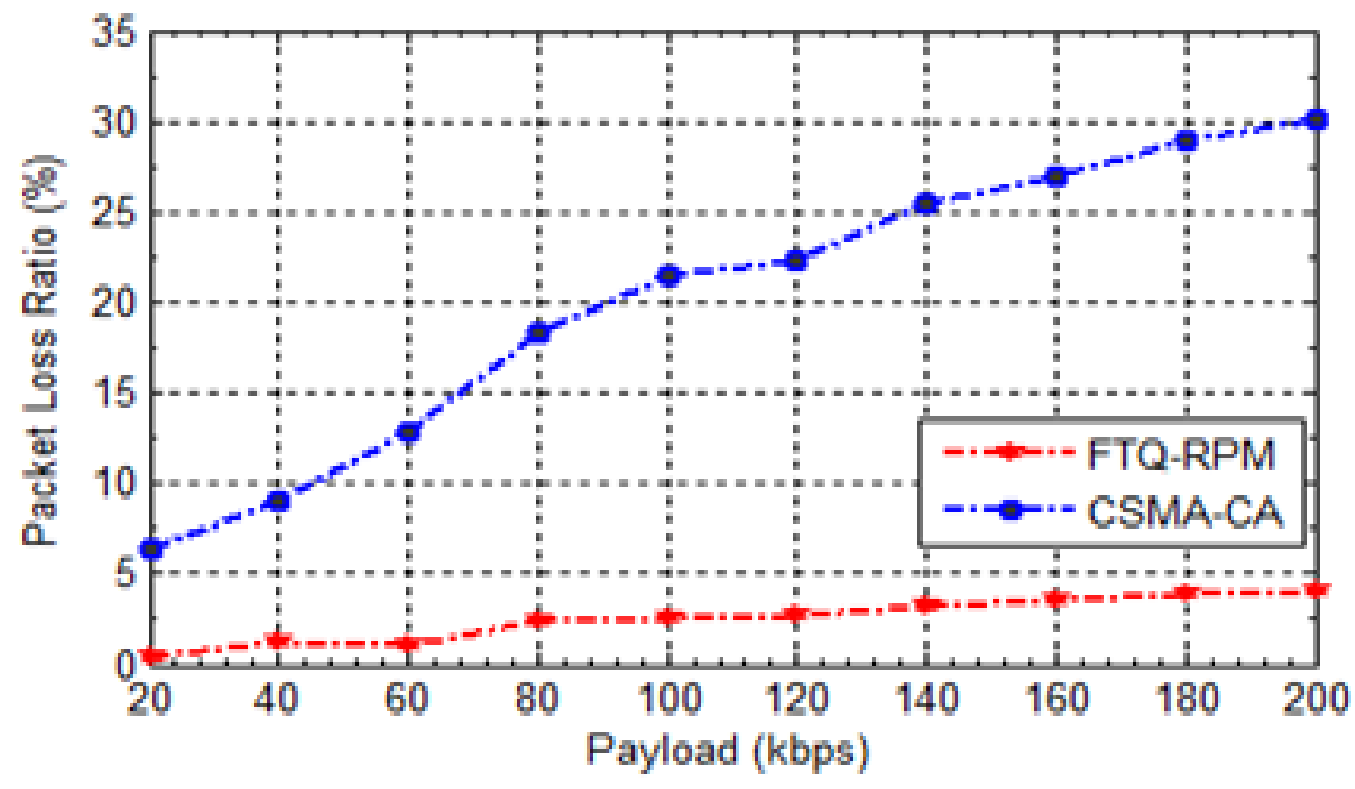

Figure 4. Packet loss variation with increase in payload. 


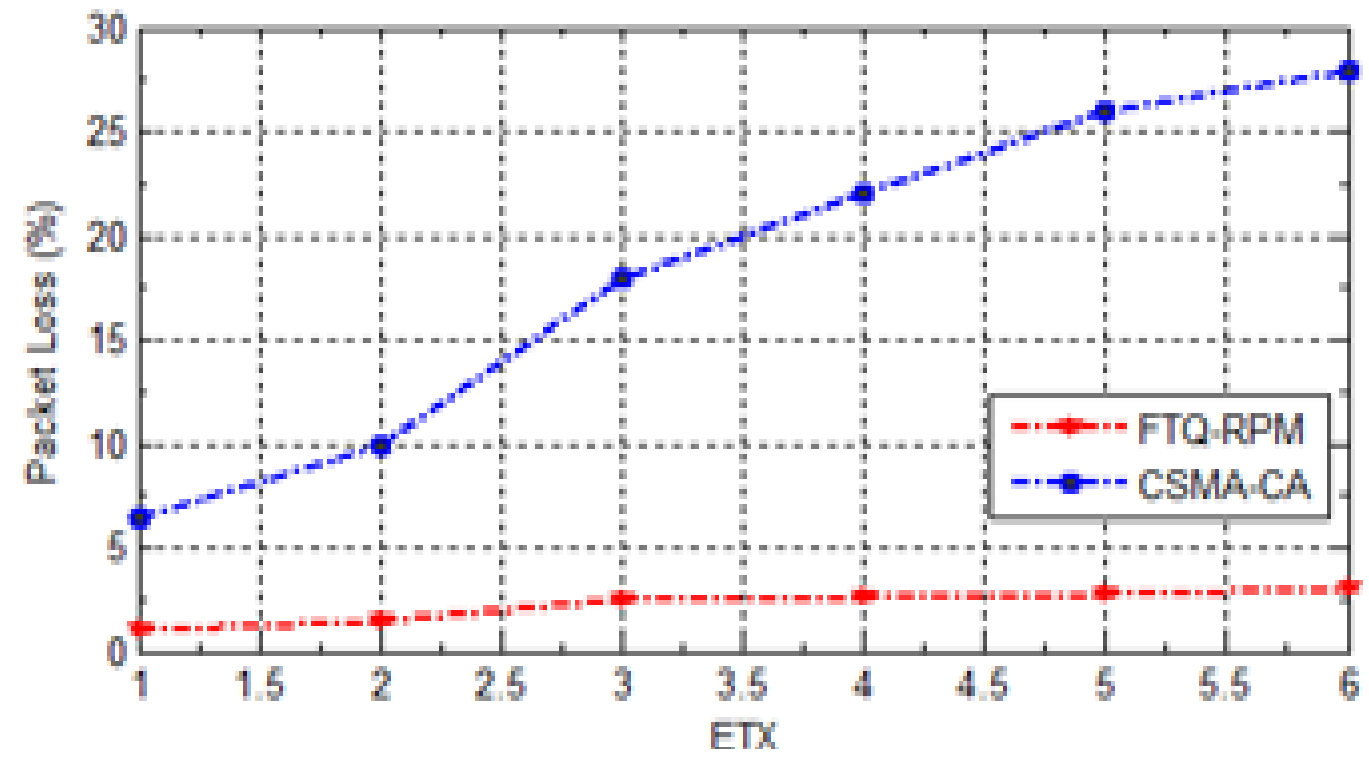

Figure 5. Packet Loss variation with increase in the number of control packets (ETX).

multimedia transmission over WMSNs, maintaining minimum number of ETX is must as it reduces the control packet's signaling overheads and delay. In FTQ-RPM the emphasis is made on reducing the ETX count while considering a node with higher RSSI to become a parent node for reliable data transmission. With these conditions, undeniably FTQ-RPM intends to provide higher throughput while maintain minimum ETX count and hence increase in ETX is not much significant. This is evident from as depicted in Figure 5, our proposed FTQ-RPM routing protocol shows almost uniform packet delivery (inverse of packet loss) irrespective of ETX counts, while on the other hand classical RPL with CSMA-CA undergoes exponentially rising packet loss over increase in ETX count. It can also be related to the fact that increase in ETX states farther inter-node distance or hop counts that

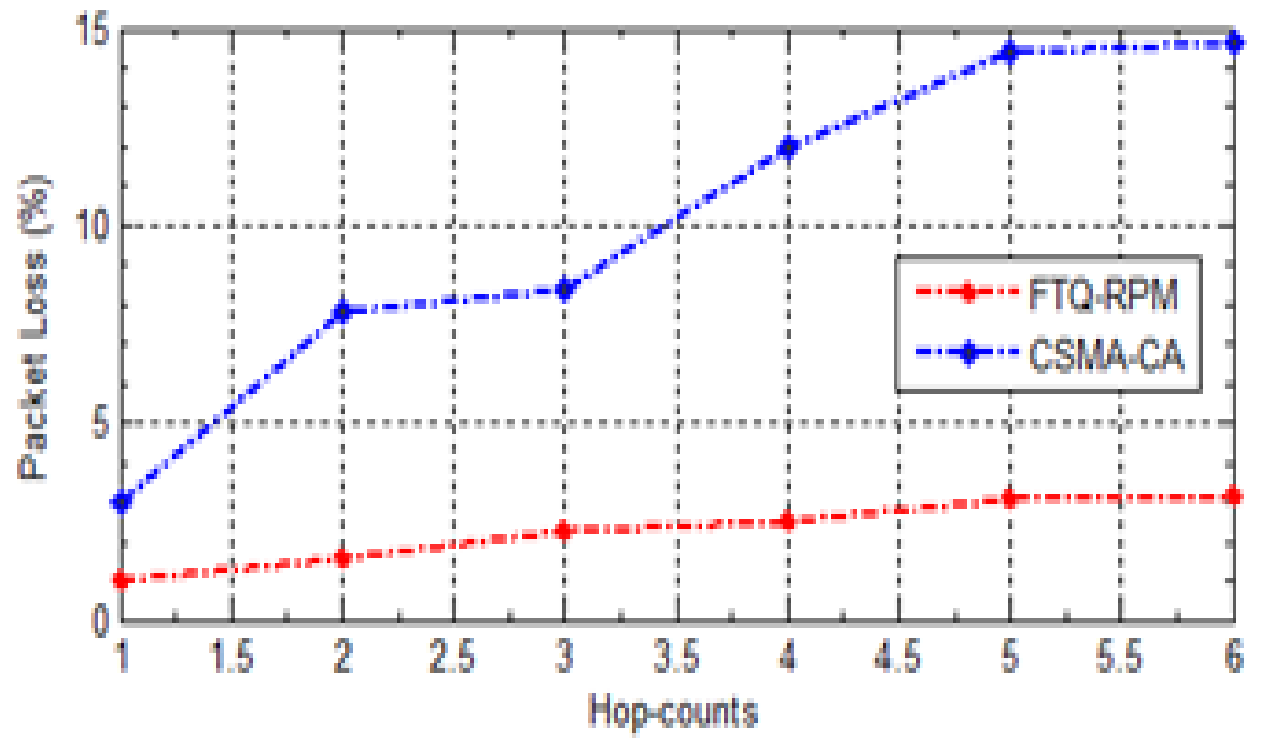

Figure 6. Packet loss variation with increase in the number of hops. 
in case of mobility might impose link-outage and hence higher data loss. This result signifies that our proposed FTQ-RPM routing protocol can assist or ensure reliable data transmission under dynamic condition even with less ETX control packets.

Figure 6 presents packet loss variation with increase in hops. As stated in above discussion that with mobile topology there could be probability of packet loss due to farther inter-node distance or node parameters changes. This as a result can cause significant packet loss. However, since our proposed model exploits node statistics such as dynamic ARSSI and EXT that shows the link reliability and stability (probability), and therefore there can be minimum number of packet loss event. Furthermore, since our proposed routing model incorporates supplementary forwarding path selection or formation and therefore ensure successful data delivery. This as a result augments the probability of successful data delivery and hence minimum packet loss (Figure6). On contrary, classical CSMA-CA based RPL protocol doesn't possess any such kind of fault-resiliency or back-up data transmission method, which causes higher packet loss as depicted in Figure 6.

To ensure QoS provision, WMSNs require delay resilient data transmission. The simulation results obtained
(Figure 7) presents the delay performance by our proposed FTQ-RPM routing protocol. Here, it can be visualized that the proposed protocol outperforms classical CSMA-CA based routing protocol for WMSNs. However, the difference between the two is low. Here, the better performance has been achieved because of minimum ETX required, fault-resilient forwarding path selection and swift supplementary path formation. These all features strengthen our proposed FTQ-RPM routing protocol to exhibit better time-efficiency.

In addition to the above mentioned performance, since our proposed routing protocol employs backup data per time slot that once detecting any link outage transmits it to the targeted recipient through supplementary path. In practice, when finding successful data delivery, our proposed FTQ-RPM routing protocol erases buffer to accommodate more data elements and hence makes it resource efficient. In such manner, our proposed routing protocol, i.e., FTQ-RPM is found efficient in terms of higher throughput, higher PDR, low loss rate, minimum delay and optimal resource utilization that cumulatively recommend it for real-time multimedia data transmission over mobile-WMSNs. Since, the proposed FTQ-RPM protocol considers all specific standards of native RPL and associated backward compatibility, suitable for being

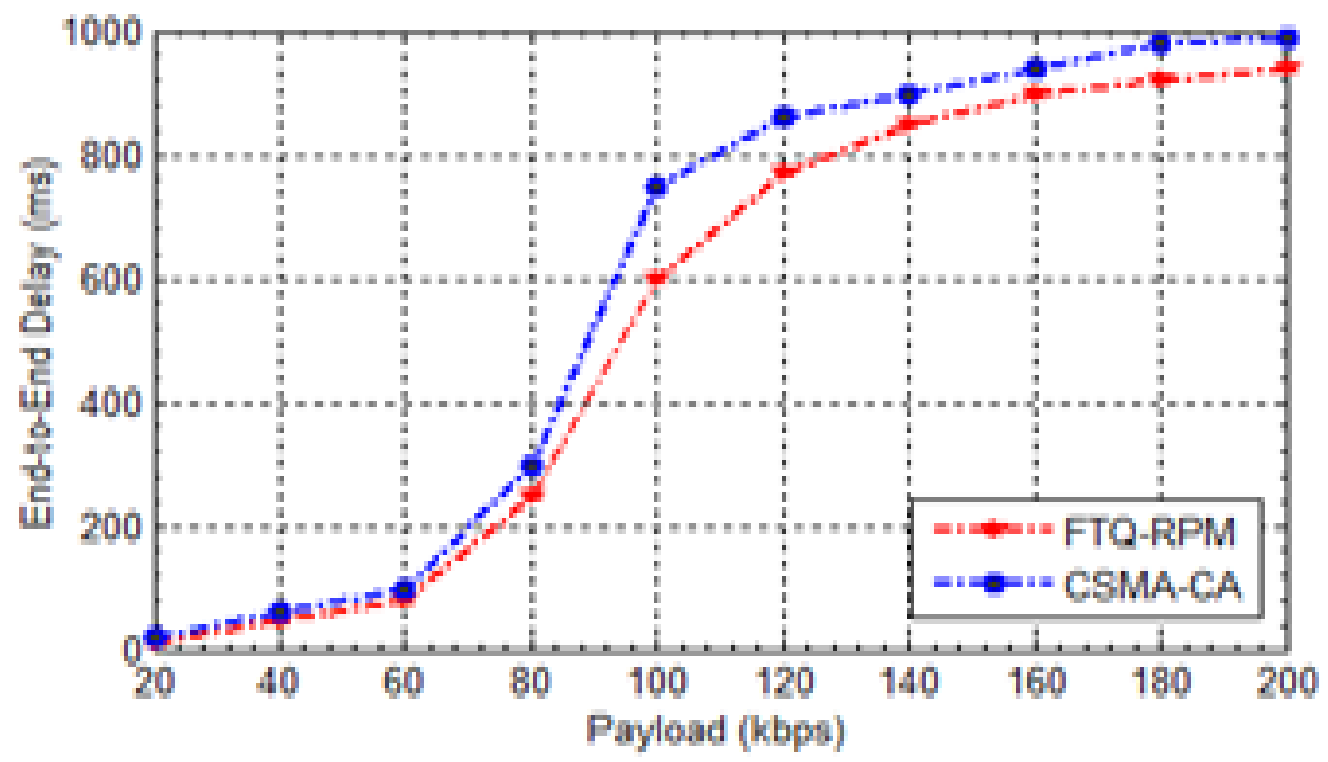

Figure 7. Delay variation with increase in payload or transmission rate. 
used in LLNs, the acceptability of our proposed routing protocol gets augmented.

\section{Conclusion}

Considering the significance of a robust and QoS-efficient routing protocol for multimedia data transmission, in this study a novel Fault-Resilient and QoS Centric Routing Protocol for WMSN (FTQ-RPM) has been developed. With intend to incorporate the proposed method with real time IoT application environment, the proposed FTQ-RPM routing protocol was developed using Contiki-Cooja platform, where the classical RPL routing protocol was augmented with mobility features and multiple network condition parameters (say, dynamic network link parameters) based parent node selection for forwarding path formation. The use of Adaptive RSSI obtained through DIO control message (a unicast message from each node as the acknowledgement of DIS for root node) enabled mobile node localization that makes overall routing fault-resilient and time efficient in data gathering, which is must for QoS delivery over WMSNs. Unlike traditional RPL protocol, FTQ-RPM applied dual objective functions; first the total number of expected control message and second the ARSSI to assess suitability of a node to become parent node. Thus, obtaining the best forwarding node the supplementary path is formed that functions in the parallel of link layer of the classical RPL. This approach enables delay-resilient supplementary path formation to transmit multimedia data in case of any link outage. To perform parent node selection swiftly this paper at first explored the parent node from a parent node set containing proactively collected nodes stored in the decreasing order of the rank matrix. It reduces computation time required for parent node selection. On the other hand, in case unavailability of the best parent node, FTQ-RPM executes node discovery and selects best parent node for supplementary path formation. The proposed method incorporates backup of the data packets which are transmitted once detecting any link outage due to mobility, and hence forming the best supplementary path the backup data is transmitted to alleviate delay and packet loss issue significantly. Thus, the overall approach where ARSSI based mobile node localization, and dual link parameter (i.e. ETX and ARSSI) based best forwarding path selection has enabled a robust multimedia data transmission system for QoS delivery over WMSNs under LLN condition. The performance assessment with varying packet rate transmission and load conditions affirms suitability of the proposed routing protocol for real time multimedia data transmission over WMSNs. In future, to augment the performance the use of multiple timers such as network discovery timers, handshake optimization related timers, link monitoring timers etc can be applied to avoid any probability of link outage and time incurred in supplementary path formation.

\section{References}

1. Akyildiz IF, Melodia T, Chowdhury KR. A survey on wireless multimedia sensor networks. Computer Networks. 2007; 51(4):921-60. https://doi.org/10.1016/j.comnet.2006.10.002.

2. Guinard D, Trifa V, Pham T, Liechti O. Towards physical mashups in the Web of Things. Proc INSS'09; 2009. p. 1-4. https://doi.org/10.1109/INSS.2009.5409925.

3. Web of Things. 2017. https://webofthings.org/2017/04/08/ what-is-the-web-of-things/

4. M. Geoff. The 6LoWPAN architecture. Proc ACM EmNets'07; 2007. p. 78-82..

5. James PF, How C, Bull D, Nix A. Distortion-based link adaptation for wireless video transmission. EURASIP Journal of Advance Signal Processing; 2008. p. 1-17.

6. Schaar MV, Turaga DS. Cross-layer packetization and retransmission strategies for delay-sensitive wireless multimedia transmission. IEEE Transaction of Multimedia. 2007; 9(1):185-97.

7. Fallah YP, Nasiopoulos P, Alnuweiri H. Efficient transmission of H.264 video over multirate IEEE 802.11e WLANs. EURASIP Journal of Wireless Communication and Networking. 2008.

8. Heinzelman W, Kulik J, Balakrishnan H. Adaptive protocols for information dissemination in Wireless Sensor Networks. Proc of the 5th ACM/IEEE Mobicom Conference (MobiCom '99); Seattle, WA. 1999. p. 174-85. https://doi. org/10.1145/313451.313529.

9. Intanagonwiwat C, Govindan R, Estrin D. Directed diffusion: A scalable and robust communication paradigm for sensor networks. Proc of the ACM MobiCom '00; Boston, MA. 2000. p. 56-67. 
10. Yao Y, Gehrke J. The cougar approach to in-network query processing in sensor networks. ACM SIGMOD Record. 2002; 31(3):9-18. https://doi.org/10.1145/601858. 601861 .

11. Heinzelman W, Chandrakasan A, Balakrishnan H. Energyefficient communication protocol for Wireless Microsensor Networks. Proc of the 33rd Hawaii International Conference on System Sciences (HICSS '00); 2000. p. 1-10. https://doi. org/10.1109/HICSS.2000.926982.

12. Lindsey S, Raghavendra C. PEGASIS: Power-Efficient Gathering in Sensor Information Systems. Proc of the IEEE Aerospace Conference. 2002; 3:1125-30.

13. Winter T, Thubert P, Brandt A, Clausen T, Hui J, Kelsey R, Levis P, Pister K, Struik R, Vasseur J. RPL: IPv6 Routing Protocol for Low power and Lossy Networks. RFC 6550, IETF ROLL WG. 2012.

14. Vasseur JP. Terminology in Low power and Lossy Networks, draftietf-roll-terminology-06.txt. 2011. p. 1-8.

15. Lee KC, Sudhaakar R, Ning J, Dai L, Addepalli S, Vasseur JP, Gerla M. A Comprehensive evaluation of RPL under mobility. Hindawi Publishing Corporation. International Journal of Vehicular Technology. 2012; 2012:1-10. https:// doi.org/10.1155/2012/904308.

16. Vasseur J, Dunkels A. Interconnecting smart objects with IP: The Next Internet, Morgan Kaufmann, 1 ed. 2010. p. 1-432.

17. Amendment 8 Medium Access Control (MAC) Quality of Service (QoS) Enhancements. 2005. https://ieeexplore.ieee. org/document/1541572.

18. Chen M, Leung VC, Mao S, Yuan Y. Directional geographical routing for real-time video communications in Wireless Sensor Networks. Computer Communication. 2007; 30(7):3368-83. https://doi.org/10.1016/j.comcom.2007.01.016.

19. Oldewurtel F, Ansari J, Mahonen P. Cross-layer design for distributed source coding in Wireless Sensor Networks. Proc SENSORCOM' 08; 2008. p. 435-43. https://doi. org/10.1109/SENSORCOMM.2008.10.

20. Amala Shiny VA, Nagarajan V. Energy efficient routing protocol for Mobile Wireless Sensor Network. International Journal of Computer Applications. 2012; 43(21):1-5.

21. Shiang HP, Var der Schaar M. Distributed resource management in multi-hop cognitive radio networks for delay sensitive transmission. IEEE Transactions on Vehicular Technology. 2009; 58(2):941-53. https://doi.org/10.1109/ TVT.2008.925308.

22. Frossard P, De Martin JC, Civanlar R. Media streaming with network diversity. Proc IEEE. 2008; 96(1):39-53. https:// doi.org/10.1109/JPROC.2007.909876.

23. Othman JB, Yahya B. Energy efficient and QoS based routing protocol for Wireless Sensor Networks. Journal of Parallel and Distributed Computing. 2010; 70(8):849-57. https:// doi.org/10.1016/j.jpdc.2010.02.010.

24. Saxena N, Roy A, Shin J. Dynamic duty cycle and adaptive contention window based QoS-MAC protocol for wireless multimedia sensor networks. Computer Networks. 2008; 52(13):2532-42. https://doi.org/10.1016/j.comnet.2008.05.009.

25. Farrag O, Younis M, Amico WD. MAC support for wireless multimedia sensor networks. Proc IEEE GLOBECOM'09; 2009. p. 3781-6. https://doi.org/10.1109/ GLOCOM.2009.5425242.

26. Kimand H, Min SG. Priority-based QoS MAC protocol for Wireless Sensor Networks. Proc IEEE IPDPS'09; 2009. p. $1-8$.

27. Schaar MV, Turaga DS. Cross-layer packetization and retransmission strategies for delay-sensitive wireless multimedia transmission. IEEE Transacton Multimedia. 2007; 9(1):185-97. https://doi.org/10.1109/TMM.2006.886384.

28. Jaradat T, Benhaddou D, Balakrishnan M, Al-Fuqaha A. Energy efficient cross-layer routing protocol in Wireless Sensor Networks based on fuzzy logic. 9th International Wireless Communications and Mobile Computing Conference (IWCMC); Sardinia. 2013. p. 177-82. https:// doi.org/10.1109/IWCMC.2013.6583555.

29. Brar GS, Rani S, Chopra V, Malhotra R, Song H, Ahmed SH. Energy efficient direction-based PDORP routing protocol for WMSN. IEEE Access. 2016; 4:3182-94. https://doi. org/10.1109/ACCESS.2016.2576475.

30. Tanwer A, Hussain M, Singh Reel P. Energy efficient wireless sensing framework to enhance mobile learning. International Conf on Tech for Education; 2010. p. 50-5.

31. Yahya B, Ben-Othman J. An energy efficient and QoS aware multipath routing protocol for Wireless Sensor Networks. Local Computer Networks, IEEE 34th Conference; 2009. p. 93-100.

32. Ben Saad L, Chauvenet C, Tourancheau B. Simulation of the RPL routing protocol for IPv6 sensor networks: Two cases studies. International Conference on Sensor Technologies and Applications SENSORCOMM; 2011. p. 1-4.

33. Ben Saad L, Tourancheau B. Sinks mobility strategy in IPv6- based WSNs for network lifetime improvement. International Conference on New Technologies, Mobility and Security (NTMS) IFIP; Paris, France. 2011. p. 1-5.

34. Korbi E, Ben Brahim M, Adjih C, Saidane LA. Mobility enhanced RPL for Wireless Sensor Networks. Third International Conference on the Network of the Future (NOF); Gammarth. 2012. p. 1-8.

35. Gaddour O, Koubaa A, Rangarajan R, Cheikhrouhou O, Tovar E, Abid M. Co-RPL: RPL routing for mobile low power Wireless Sensor Networks using Corona mechanism. 
Proceedings of the 9th IEEE International Symposium on Industrial Embedded Systems; 2014. p. 200-9. https://doi. org/10.1109/SIES.2014.6871205.

36. Somaa F, Korbi IE, Adjih C, Saidane LA. A modified RPL for Wireless Sensor Networks with Bayesian inference mobility prediction. International Wireless Communications and Mobile Computing Conference (IWCMC); 2016. p. 690-5. https://doi.org/10.1109/IWCMC.2016.7577140.

37. Lohith YS, Narasimman TS, Anand SVR, Hedge M. Link Peek: A link outage resilient IP packet forwarding mechanism for 6LoWPAN/RPL based Low power and Lossy Networks (LLNs). IEEE International Conference on Mobile Services; 2015. p. 65-72. https://doi.org/10.1109/ MobServ.2015.19.

38. Lee C. RPL under mobility. Proceeding of the IEEE Consumer Communications and Networking Conference (CCNC). 2012.
39. Gungor VC, Hancke GP. Industrial Wireless Sensor Networks: Applications, protocols, and standards. CRC Press, Inc., Boca Raton, FL, USA, 1st ed. 2013.

40. Raman B, Chebrolu K, Madabhushi N, Gokhale DY, Valiveti $\mathrm{PK}$, Jain D. Implications of link range and (in) stability on sensor network architecture. WiNTECH '06, ACM; New York, USA. 2006. p. 65-72.

41. Srinivasan K, Levis P. Rssi is under appreciated. Proceedings of the Third Workshop on Embedded Networked Sensors, EmNets; 2006.

42. Farooq O, Kunz T. Contiki-based IEEE 802.15.4 channel capacity estimation and suitability of its CSMA-CA MAC layer protocol for real-time multimedia applications. Hindawi Publishing Corporation Mobile Information Systems. 2015; 2015:1-9. PMid: 25625082 PMCid: PMC4296390. 